Rabaska

Revue d'ethnologie de l'Amérique française

RABASKA

\title{
Marius Barbeau, en quête de connaissances... depuis 1911
}

\section{Benoît Thériault}

Volume 9, 2011

URI : https://id.erudit.org/iderudit/1005900ar

DOI : https://doi.org/10.7202/1005900ar

Aller au sommaire du numéro

Éditeur(s)

Société québécoise d'ethnologie

ISSN

1703-7433 (imprimé)

1916-7350 (numérique)

Découvrir la revue

Citer ce document

Thériault, B. (2011). Marius Barbeau, en quête de connaissances... depuis 1911. Rabaska, 9, 165-181. https://doi.org/10.7202/1005900ar

\section{Résumé de l'article}

En 1945, Marius Barbeau, pionnier des études folkloriques au Canada, donnait son premier cours à la chaire de folklore de l'Université Laval, nouvellement créée. Pour lui, c'était l'aboutissement de près de 35 années de travail de terrain et d'efforts pour faire reconnaître le folklore. Ce cours, intitulé « En quête de connaissances anthropologiques et folkloriques dans l'Amérique du Nord depuis 1911 ", dresse un bilan de sa carrière, expose ses théories et sa conception du rôle du folklore et traite, sous forme de conseil, de sa méthodologie de recherche. Ce sont ces conseils, provenant de la section de son cours « Comment on recueille les contes, les chants, les mélodies, le langage, les spécimens ou les données s’y rapportant, parmi les Sauvages ou les Blancs ", que ce document fait revivre. 


\title{
Inédit
}

\section{Marius Barbeau, en quête de connaissances... depuis 1911}

\author{
PRÉSENTÉ PAR BENOÎT ThÉRIAUlT \\ Musée canadien des civilisations, Gatineau
}

L'année 2011 marque le centenaire de l'entrée en fonction de Marius Barbeau, premier anthropologue canadien de formation, à la division d'anthropologie de la Commission géologique du Canada (aujourd'hui le Musée canadien des civilisations). Créé en 1910 suite aux pressions soutenues de la Société royale du Canada et de la British Association for the advancement of Science ${ }^{1}$, ce premier centre d'étude anthropologique canadien était alors à la recherche de candidats pour combler les quelques postes disponibles.

Candidat pressenti pour 1'un de ces postes, Barbeau revenait tout juste d'Oxford où il avait obtenu un diplôme en anthropologie, une première pour un Canadien ${ }^{2}$. C'est donc muni de ce diplôme et avec en main une lettre de recommandation de sir William Osler que Barbeau se présente à l'automne 1910, à Ottawa, au bureau du directeur de la Commission géologique, Reginald W. Brook. Barbeau arrive toutefois trop tard pour se voir offrir

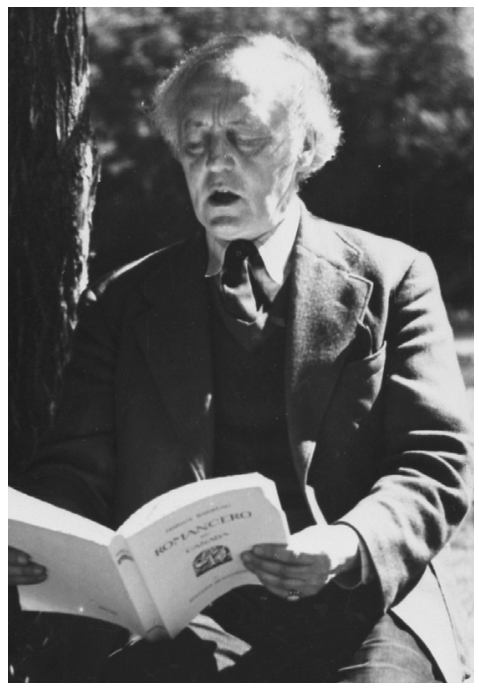

Marius Barbeau assis sous les arbres lisant un extrait de son Romancero du Canada (1937) à un groupe d'étudiants [vers 1945]. Musée canadien des civilisations, négatif 86-1252.

1. Le fruit était mûr depuis plusieurs années. Déjà, en 1856, la Commission géologique du Canada avait eu le mandat de créer un premier musée. Plusieurs géologues dont George Dawson avaient pour habitude de recueillir des informations auprès des Amérindiens. Dawson avait ainsi publié dans son rapport de 1878-1879 un remarquable document sur les caractéristiques, les traditions et la langue des Haïdas des îles de la Reine Charlotte en Colombie-Britannique.

2. En fait, doté d'une bourse Rhodes, une première pour un universitaire canadien-français, Barbeau prévoyait y faire des études de droit. Mais, ayant peu d'intérêt pour ce domaine et sous les recommandations de son tuteur à Oriel College, le révérend L.R. Phelps, il devait s'inscrire en anthropologie sous la direction de Robert Ranulph Marett. 
le poste de directeur de la nouvelle division. Brook, sous les recommandations de Franz Boas de la Columbia University, venait d'engager à ce titre, le 3 septembre 1910, un Américain, Edward Sapir, ancien étudiant de Boas. Sapir était déjà parti en mission chez les Nooktas de la Colombie-Britannique et Barbeau dut attendre son retour pour être embauché. Ce fut finalement le cas à la fin de décembre 1910, et Barbeau fit ses débuts le 2 janvier $1911^{3}$.

À la mi-avril 1911 Barbeau amorce sa première enquête anthropologique chez les Hurons de Lorette (aujourd'hui Wendake) près de Québec. Curieusement, c'est là que, pour la première fois, il est mis en contact, du moins consciemment, avec le folklore. Ainsi, au cours de ses séances de travail avec ses informateurs hurons, on lui propose des contes qui, bien qu'intégrés dans la culture orale huronne, sont à l'évidence d'origine française : «La Princesse de la jarretière verte », « Ti-Jean et les géants », «L'Eau de la fontaine de Paris " $^{4}$. À ce moment-là, il n'est nullement question d'études folkloriques, le mandat de la division d'anthropologie étant strictement limité aux études autochtones. Aussi, Barbeau refuse-t-il de les recueillir. Toutefois, plus tard en décembre 1913, lors d'une réunion de l'American Anthropological Association à New-York, le professeur Franz Boas, qui s'intéressait à la pénétration des contes européens chez les Amérindiens des États-Unis, interrogea Barbeau sur la question : « The Indian as far as Mexico have folktales that can be only French in their origin [...]. There are so many French-Canadian "coureurs des bois", could they not have transferred some of their stories to them $?^{5} \gg \mathrm{Ne}$ sachant trop quoi répondre, Barbeau fit part à Boas des contes qu'on lui avait proposés à Lorette. Sur ce, Boas enjoint Barbeau de retourner à Lorette pour y obtenir le plus d'informations possible, lui proposant du même coup de devenir éditeur associé du Journal of American Folk-Lore dans lequel il s'engage à publier en français des numéros sur le folklore canadien-français ${ }^{6}$. Pour Barbeau, ce sera là l'élément déclencheur et le début de sa longue carrière de folkloriste. Au cours de l'été 1914, Barbeau retourne donc à Lorette où il lance ses quêtes folkloriques sans toutefois négliger son travail d'ethnologue en études amérindiennes. Rapidement, il oriente ses recherches du côté de Charlevoix, de la Beauce, du Bas-Saint-Laurent et de la Gaspésie où la cueillette se montre abondante. En septembre 1917, il fait la rencontre d'Édouard-Zotique

3. Barbeau demeurera toute sa carrière à l'emploi du Musée mémorial Victoria connu par la suite comme le Musée national du Canada. Même après sa retraite le 5 mars 1949, il continuera d'y travailler et cela jusqu'au moment de son décès survenu le 27 février 1969.

4. Laurence Nowry, Marius Barbeau, Man of Mana, Toronto, NC Press, 1995, p. 141.

5. Ibid.

6. Boas envisageait de fait la création d'une succursale canadienne de l'American Folk-Lore Society, qui prendrait charge de ces numéros spécifiquement canadiens ( $c f$. Musée canadien des civilisations [Mcc], Archives, lettre de Franz Boas à R.W. Brook, 14 janvier 1914. Correspondance d'Edward Sapir, dossier « Boas, Franz, 1910-1914 » boîte 621 f.1). 
Massicotte avec qui commence une longue collaboration. Conscient que les études folkloriques ne font pas partie à proprement parler du mandat de la division d'anthropologie, Barbeau n'a d'autre choix que de se montrer persuasif. Fort de l'appui de Boas, il met sur pied en 1915 une division canadienne de l'American Folk-Lore Society qui, en 1917, se divise en succursales ontarienne et québécoise ${ }^{7}$. Dans l'une comme dans l'autre, il recrute à titre d'organisateurs des membres de son entourage, tant social que professionnel, de même que ses relations politiques ${ }^{8}$. Plusieurs membres de la division d'anthropologie s'y retrouvent conscrits, incluant, à son corps défendant, Edward Sapir qui n'a d'autre choix que d'appuyer ce projet cher à son mentor Franz Boas. Au nombre des premiers membres recrutés par Barbeau, on retrouve le célèbre sir Wilfrid Laurier : "Ottawa, 14 octobre 1916. [...]. J'accepte avec plaisir l'invitation de devenir membre de "The American Folk-Lore Society" $»$. Rien de surprenant, pour cette œuvre que l'on présentait comme étant «à la fois scientifique et nationale». En mai 1922, les deux divisions provinciales sont fondues en une seule, pancanadienne, qui subsistera jusqu'en décembre 1948.

$\mathrm{Au}$ cours de cette période Barbeau profitera pleinement de cette formidable vitrine que lui offre cette organisation tout d'abord par sa revue spécialisée où il lui est possible de publier en français, mais aussi en organisant de 1918 à 1922 à Montréal, à Québec et à Ottawa, des soirées et concerts folkloriques ouverts à tous les publics dont un en 1922 aux écoliers d'Ottawa. Pour lui toutes les tribunes seront bonnes pour faire la promotion du folklore : publications scientifiques, publications populaires parfois romancées, articles dans des revues d'associations professionnelles de tous genres, des magazines à grand tirage, des journaux incluant les pages enfantines, des revues destinées aux jeunes, le tout tant en français qu'en anglais. À cela s'ajoutent des conférences publiques et des conférences spectacles où il se fait accompagner d'un chanteur, d'un conteur ou d'un artisan du terroir. Sans compter les fameux festivals de folklore de Québec de 1927 et 1928 organisés par le Canadien

7. L'idée de créer deux succursales permet un double recrutement, mais surtout de s'adresser à deux milieux différents. L'organisation, qui s'affiche sous le nom de "Société américaine de folklore » au Québec, reçoit, l'appui du gouvernement du Québec qui est responsable de 100 souscriptions distribuées aux députés du parlement, à certains hauts fonctionnaires de même qu'aux directeurs d'écoles normales de la province. Cet appui indéfectible sera renouvelé par les gouvernements de toutes allégeances jusqu'à la fin en 1948. À cela, s'ajoutent la société Saint-Jean Baptiste puis, à titre personnel, des membres du clergé, des notables (avocats, notaires, médecins), des enseignants et certains individus.

8. Barbeau fait partie de cette élite, tant francophone qu'anglophone du pays, qui gravite à Ottawa. Fait membre de la Société royale du Canada en 1916, il multiplie ses relations et s'ouvre ainsi bien des portes qu'il n'hésitera pas à traverser.

9. Mcc, Archives, lettre de Wilfrid Laurier à Marius Barbeau, 14 octobre 1916. Correspondance de Marius Barbeau « Laurier, sir Wilfrid (1916) », boîte B212 f.20. 
Pacifique $^{10}$. Enfin Barbeau ne néglige pas la radio où tout au long de sa carrière il sera fréquemment invité de même que plus tard à la télévision ${ }^{11}$.

Barbeau avait donc pris tous les moyens possibles pour faire connaître le folklore principalement canadien-français. Mais, au début des années 1940, l'effort de guerre mobilisait toutes les ressources. Le Musée, sans être fermé, n'avait plus de budget pour la recherche. Pendant un certain temps, Barbeau fut même réaffecté aux services des pensions pour veuves des soldats. Bien que l'avenir semblât incertain, le synchronisme des événements permit à Barbeau de s'investir dans un nouveau champ d'activité, celui de l'enseignement. Il était temps d'élever les études folkloriques au Canada au rang de science, et de les proposer à l'université. Il était temps également, sans doute, de penser à la relève ${ }^{12}$. C'était là l'aboutissement logique du travail effectué depuis les débuts, comme il l'indiquera en 1945 dans une note au directeur du Musée, F.C.C. Lynch :

Educational development in anthropological and folklore studies is the natural outcome of the work carried out for many years by the National Museum. Indeed, it has taken 30 years to prepare the ground. Now our efforts are beginning to bear fruit. The universities intend, under our guidance and tuition, to form young ethnologists and folklorists. They are much needed ${ }^{13}$.

Déjà, depuis 1942, Barbeau avait inauguré une série de cours à l'Université d'Ottawa sous le titre de « Human Geography of North America » et portant sur l'anthropologie amérindienne. De même, à l'invitation de Luc Lacourcière, il donnait des conférences sur le folklore dans le cadre des cours d'été de la faculté des lettres de l'Université Laval. Il prêta ainsi son concours à Lacourcière et à $\mathrm{M}^{\mathrm{gr}}$ Félix Antoine Savard dans leurs efforts pour obtenir la création, à l'Université Laval, d'une chaire de folklore, dont il devait être le garant, voire la caution scientifique, ainsi qu'il l'exprimait au directeur du Musée F.C.C. Lynch :

I have been told, more or less confidentially, that Hon. Onesime Gagnon, Provincial Treasurer, has mentioned my appointment to a professorship as a

10. En 1927 et 1928, John Murray Gibbons, agent de publicité du Canadien Pacifique, avait confié à Marius Barbeau la programmation du « Festival de la chanson et des métiers du terroir » tenu au Château Frontenac de Québec. Bénéficiant d'importants moyens financiers et d'une grande liberté, Barbeau y exprima sa large vision du folklore et en fit un véritable succès.

11. Selon Laurence Nowry, Barbeau aurait dès 1926 donné une entrevue radiophonique portant sur le folklore canadien-français à Toronto sur les ondes de CKCL (cf. Nowry, op. cit., p. 304).

12. Pour lui-même, il avait songé à Luc Lacourcière, puis à son gendre Marcel Rioux qui travaillera au Musée un certain nombre d'années (1947-1959), enfin à Carmen Roy sous l'impulsion de qui le folklore fera sa place au Musée national de l'homme, en devenant section en 1957, division en 1966 et enfin en juin 1970, grâce à ses initiatives de recherche sur les cultures des groupes ethniques canadiens, le « Centre canadien d'études sur la culture traditionnelle».

13. Note de service de Marius Barbeau à F.C.C. Lynch, Ottawa, 10 avril 1945. Mcc, Archives, fonds Marius Barbeau, correspondance, dossier « Lynch, F.C.C. (1944 - 1946) », boîte B216 f.14. 
prerequisite for granting a government appropriation to the university for the study of folklore and ethnography ${ }^{14}$.

Aussi, lorsqu'en février 1944 cette chaire fut officiellement fondée, Barbeau était prêt. Il avait déjà en main des notes de cours dactylographiées, accompagnées de notes manuscrites destinées à enrichir son enseignement. Après certains tâtonnements, Barbeau put enfin lancer ses cours le 8 mars 1945. Sous le titre En quête de connaissances anthropologiques et folkloriques dans l'Amérique du Nord depuis 1911, son programme se distribuait en 45 leçons (incluant six conférences publiques) divisées en trois séries devant être dispensées aux sessions du printemps 1945, de l'automne 1945 et du printemps $1946^{15}$. Les cours se donnaient en rafale pendant trois semaines, pratiquement tous les jours, sous le coup de $17 \mathrm{~h}$, à raison d'une heure par leçon. Les conférences publiques (les Mardis universitaires) avaient lieu deux fois par session, les mardis à compter de $20 \mathrm{~h} 15$.

Pour la première fois, Barbeau pouvait, non plus simplement faire connaître le folklore, mais former des spécialistes qui, à leur tour, pourraient poursuivre et approfondir les nombreux champs d'études qu'il avait ébauchés depuis 1911, des champs d'études interreliés incluant les études amérindiennes, à l'image de sa propre expérience.

C'est d'ailleurs l'intention de vos directeurs et de vos maîtres à l'université d'éveiller chez vous le goût des recherches anthropologiques, et de préparer du moins quelques vocations de folkloristes, d'ethnographes, d'archéologues et de linguistes. Nos universités, nos musées et nos institutions en ont déjà besoin, mais ils ne sauraient trouver chez nous que quelques rares devanciers. Les connaissances de ce genre à l'avenir seront plus utiles que jamais, car le Canada cesse rapidement d'être une colonie de l'Europe ; il se suffira bientôt à luimême. Mais pour cela il lui faut ses lumières en propre, et les connaissances que je vous propose ici sont essentielles à la vraie indépendance ${ }^{16}$.

Barbeau avait ainsi divisé son cours en trois séries. La première série donnée en mars 1945, se voulait une présentation des «milieux géographiques et ethniques » du Canada de même qu'un bilan de la recherche anthropologique au Canada, de ses expériences personnelles de recherche en études amérindiennes et de sa découverte du folklore. La deuxième série, donnée en octobre 1945, constituait un ensemble de conseils pratiques aux futurs

14. Ibid.

15. Au départ son cours prévoyait 30 leçons, mais finalement il fut prolongé pour couvrir trois semestres.

16. Marius Barbeau, «En quête de connaissances anthropologiques et folkloriques dans l'Amérique du Nord depuis 1911 », Résumé d'un cours donné à la Faculté des Lettres, mars-octobre 1945, Québec, Archives de folklore, Université Laval, 1945, [83 p.] p. 2. Mcc, Archives, fonds Marius Barbeau, Dossier «En quête de connaissances anthropologiques et folkloriques... : Résumé de cours », boîte B356 f.10. 
folkloristes et ethnologues, basé sur une expérience de près de 35 ans de terrain. La troisième série, donnée au printemps 1946, est essentiellement théorique et passe en revue les grandes écoles d'anthropologie française, anglaise et étatsunienne, puis traite du rôle des études anthropologiques au point de vue scientifique, culturel et au point de vue de la nationalité.

Cet enseignement est important à plusieurs égards : il est le premier cours dispensé par Barbeau à la chaire de folklore de Laval. Le maître y fait le bilan de sa carrière et expose pour la première fois sa méthodologie de recherche, ses théories et sa conception du rôle du folklore dans la perspective du développement d'une identité et d'une culture nationales. Il est aussi intéressant par le ton adopté par Barbeau qui évoque une époque en marquant la distance entre le chercheur, celui qui étudie, et son informateur, qui est le sujet de son étude, sans y attacher le moindre élément de supériorité. Enfin, ce cours ne sera jamais repris par Barbeau, qui, durant les années suivantes, se consacrera à des thèmes particuliers : «Folklore canadien (chanté, dansé, raconté, dit, brodé, ouvré, sculpté, peint, goûté et bu)» en 1946-1947; ou « La chanson populaire en France et par le monde » au printemps 194917.

Pour toutes ces raisons, nous avons pensé honorer la mémoire du « vieux maître » en cette année du centenaire de ses débuts et saluer les générations de chercheurs qui, à la suite de Marius Barbeau, ont fait progresser notre quête de connaissances anthropologiques et folkloriques. Afin de faire revivre, en partie du moins, ce premier enseignement voué à la formation de folkloristes et d'ethnologues, aboutissement logique du travail accompli depuis 1911, nous avons choisi un extrait des leçons qu'il livrait en octobre 1945 et qui porte sur la cueillette du " patrimoine immatériel » dans son véritable sens : « Comment on recueille les contes, les chants, les mélodies, le langage, les spécimens ou les données s’y rapportant, parmi les Sauvages ou les Blancs ${ }^{18} »$.

17. Barbeau donnera des cours à l'Université Laval jusqu'un 1954. Il donnait également en parallèle des cours d'anthropologie puis de folklore à l'Université de Montréal (1945-1948) de même que des cours d'anthropologie et de géographie humaine à l'Université d'Ottawa (1942-1950). Dans tous les cas, il agissait à titre de professeur invité et, selon les cas, s'absentait du Musée pour quelques semaines en utilisant parfois ses vacances ou en jumelant ses cours à des activités de recherche. Quoi qu'il en soit, il devait toujours obtenir la permission du directeur du Musée.

18. Marius Barbeau, «En quête de connaissances [...]», op. cit., p. 43-52. 


\section{Comment on recueille les contes, les chants, les mélodies, le langage, les spécimens ou les données s'y rapportant, parmi les Sauvages ${ }^{19}$ ou les Blancs}

Marius Barbeau

Musée national du Canada, Ottawa

En vue des recherches ethnographiques ou folkloriques, il faut se munir du matériel nécessaire à la cueillette ; ce matériel varie suivant le sujet à étudier. Une fois à l'œuvre, il faut bien savoir comment exécuter le travail, à l'aide de ces instruments.

\section{Instruments, outillage, cahiers}

Les études d'anthropologie physique et d'anthropométrie, par exemple, requièrent des instruments particuliers à ces sciences. Qui les a utilisés au laboratoire les connaît parfaitement ; ils sont fabriqués pour les spécialistes, en vue d'un usage particulier. Cela est aussi vrai pour les archéologues au cours de leurs fouilles minutieuses. Le folklore et l'ethnographie opèrent leurs recherches sur les vivants; leur outillage dépend du sujet choisi au préalable.

D'abord, les chercheurs ont besoin de cahiers de petit format qu'ils gardent à leur portée, dans la poche d'un habit. Comme ils doivent voyager les mains libres, au beau temps, à la pluie, en voiture, à bicyclette, en bateau, en canot, ces cahiers doivent être protégés par une bonne couverture. Au Musée national, on se sert de cahiers de 50 pages avec douille à crayon; de deux grandeurs - les petits pour la poche d'habit en voyage, les plus grands pour travail à l'atelier.

Stylos, plume, crayons, encre. [Edward] Sapir se servait de crayon indélébile. Moi-même j'emploie un stylo en expédition, la plume chez moi. Le stylo est indispensable en voyage, surtout pour la sténographie. L'inconvénient du crayon est que la pression prolongée fatigue et l'écriture

19. Tout au long de son texte. Marius Barbeau utilise le terme «sauvage» pour désigner les Amérindiens. Ce terme, aujourd'hui heureusement disparu, était encore en usage il y a 65 ans. Pour sa part, Barbeau n'y attachait pas de signification péjorative. Aussi l'avons-nous conservé par souci d'authenticité. 
s'embrouille. L'écriture doit être aussi claire que possible, surtout dans l'enregistrement de mots peu connus ou de textes en langue étrangère, qui risquent d'être relus longtemps plus tard ou qui le seront par d'autres.

Phonographe et accessoires y compris les disques pour l'enregistrement des airs et de spécimens de langage. La musique s'enregistre bien sur le phonographe ; le langage plus imparfaitement ; des échantillons, toutefois, sont utiles.

Caméras. Il est mieux de ne pas s'encombrer d'appareils lourds compliqués et facilement détraqués. L'économie d'effort, de pratique, et la continuité dans les séries photographiques sont recommandables. L'expérience dans l'art de la photographie est presque essentielle. En photographie, l'artiste compte plus, à vrai dire, que l'appareil. Le vulgaire dénote peu d'entendement lorsqu'il s'exclame : « Quel bon appareil vous possédez ! Vous faites de si bonnes photographies!» L'appareil, sans doute, doit être de bonne qualité, mais savoir en tirer parti est bien plus important. C'est l'œil du chercheur et le choix du sujet qui comptent. Par exemple, comment doit-on photographier un Sauvage ou un habitant, ou un artisan intéressant ? Du point de vue anthropométrique $?^{20}$ En tant qu'artisan ou homme de travail ? Il faut décider suivant le cas. Si vous choisissez un artisan, photographiez-le à la besogne, dans sa boutique ou au chantier ; un habitant, à sa charrue ou à sa faux ; une fileuse à son rouet. C'est déguiser un peu la vérité que de les laisser s'endimancher, comme on le faisait dans les anciennes photographies. Les plus utiles photos sont celles où l'on observe un procédé manuel qui, à défaut de discernement de l'observateur, passerait inaperçu.

Si vous photographiez une œuvre d'art, un calice, une broderie, une sculpture, un groupe de gens en action, il faut se tenir le plus près possible de son objet, pour bien l'observer ; il faut bien placer la lumière et les ombres, mouler en quelque sorte son sujet, pour faire ressortir les traits caractéristiques. L'appareil ne reproduit que ce que votre œil a bien saisi.

Les petits objets - argenterie, broderie, etc. - doivent être photographiés de très près. À cette fin, on se sert de "portrait attachement "- de 30" à 5 ' - et on mesure la distance. Quant au reste - foyer, diaphragme, temps de l'exposé, c'est l'expérience qui sert de guide. Et il en faut beaucoup, la lumière variant suivant les saisons, la latitude, la température, l'heure du jour. Un appareil perfectionné subit l'influence de tout. Après quelque temps, l'homme et la machine s'harmonisent, au point qu'un photographe sait s'il a réussi ou s'il a raté son effet - même avant le développement de sa pellicule.

20. Les premières photographies prises par Barbeau en 1911 et 1912 chez les Hurons et les Wyandottes sont en bonne partie de ce type, soit des portraits de face et de profil. Barbeau semble avoir rapidement abandonné ce type de photographie pour se concentrer sur celles documentant les gens dans leur milieu et leur savoir-faire. 
Autres instruments. Mesures - pieds en ruban, pied-de-roi. Chaque objet observé - argenterie, sculpture, objet antique - doit être mesuré, au moins dans une de ses dimensions, souvent en tous sens, et les proportions doivent être notées, ou sur le rouleau des pellicules ou dans un cahier réservé aux photographies.

Accessoires utiles, indispensables. Des cartes géographiques sont souvent nécessaires. Si vous étudiez des Sauvages, leurs domaines de chasse, par exemple, il vous faudra des cartes ou des «blue prints », ce que vous pouvez d'ordinaire vous procurer dans certains services fédéraux ou provinciaux. Si vous avez à copier des dessins traditionnels de broderie, d'argenterie, de gravures, il est bon d'avoir du papier transparent « à tracer ». Un assortiment de crayons de couleurs, d'encre de chine, est souvent utile. Des étiquettes servent à poser sur le spécimen acheté un numéro correspondant à celui du catalogue. Des petites cartes «index » contiennent les numéros et la description des chansons recueillies au phonographe. Il est bon d'avoir une liste de tous les objets requis pour une expédition, qui sert à chaque fois ; une telle liste prévient les oublis. Le cas du Karluk et de la Can[adian] Arctic Expedition...; ; les articles qui manquaient ou qui, mal placés, se retrouvaient difficilement. À Nome [Alaska], on avait en mains les armes, mais on eut peine à retrouver les munitions ! Quant aux appareils, on doit s'assurer, avant le départ, qu'ils sont en bon état.

\section{Manière d'exécuter le travail, au cours de recherches chez les Sauvages ou les Blancs}

\section{Préparation}

Des connaissances préparatoires des sujets à découvrir et à étudier sont essentielles. Le chercheur, au cours de sa carrière, doit souvent se préparer à l'observation parfaite d'un domaine nouveau pour lui. Une expédition en pays éloigné ou rarement observé oblige à couvrir beaucoup plus de terrain qu'en territoire souvent parcouru ou facile à atteindre. Pendant l'expédition «Can[adian] Arctic, 1913 », Jenness eut recours à ses études d'université pour l'anthropométrie, l'archéologie et l'ethnologie. Mais d'ordinaire, aujourd'hui, la tendance porte à la spécialité. La qualité du travail et les résultats obtenus, au cours de recherches, dépendent du soin et de la science qu'on met à la besogne, à l'annotation, à l'enregistrement. Exemple de la cueillette de spécimens et de renseignements du Cap[itaine] Emmons en Alaska, surtout dans sa collection pour l'Amer[ican] Museum of Natural History. Pour qu'une cueillette soit de haute qualité, pour qu'elle puisse être conservée pour ainsi dire en entrepôt, pour qu'elle devienne abondante en peu de temps, il est nécessaire que le chercheur fasse preuve d'industrie, 
d'adresse et d'expérience graduellement acquise dans l'usage d'une méthode sûre. Avec de l'expérience et du doigté, à la lumière d'idées précises sur l'objet recherché, on ne peut tarder d'obtenir le fruit d'utiles enquêtes.

\section{Préliminaires}

Le choix de l'objet des recherches et de l'endroit où les poursuivre constitue le premier pas. Le flair et les renseignements peuvent aider dans ce choix, ainsi que les circonstances. Dans le cas d'études à fond d'un district, comme dans mes études des Tsimsyams, c'est le fait géographique qui domine le plan des recherches. S'il ne s'agit que de cueillettes fragmentaires, de types intéressants, il convient de trouver les districts où les matières abondent et sont représentatives.

\section{Habitudes fixes et procédés manuels dans le travail}

Il est important de toujours exécuter les opérations mécaniques de la même manière, afin d'arriver à les reproduire automatiquement, sans même y penser. Cela prévient les oublis, la confusion, dégage la pensée, l'attention, pour d'autres emplois - « The rule of thumb » anglais. Ainsi :-Comment se servir des aiguilles du Standard Edison ou d'autres appareils dans l'enregistrement ou dans la reproduction des chants et des mélodies. - Comment faire fonctionner la caméra : diaphragme, foyer, exposition ; cela indépendamment de la connaissance qu'on doit avoir des distances, de la lumière. - Comment mesurer les objets : hauteur, largeur, profondeur. - Comment noter la source des contes, des chansons : à la fin de chaque numéro, toujours demander de qui cela provient, quand, où ? Un oubli peut nuire à la valeur documentaire du morceau.

\section{Concentration et continuité - être toujours prêt}

Le temps est précieux, dans le rendement des recherches. Il n'y a que tant d'heures, de jours, ou de semaines, pour l'ensemble des reconnaissances. Chaque moment compte ; il peut être utilisé. C'est de l'économie d'argent, d'énergie, de production. Avant de partir, plutôt le soir veille du départ, de bonne heure autant que possible, il faut tout préparer, crayons, cahiers, appareils. La caméra doit être armée de sa pellicule, les lentilles nettoyées et placées dans l'appareil ; celles de remplacement doivent être accessibles. Ennuis, perte de temps subis, par exemple, lors de l'expédition pour l'Office national du film, en 1941 ([Laura] Boulton); pourtant le personnel était professionnel. Le matin il fallait réveiller les autres, les mettre sur pied ; on prenait beaucoup trop de temps à déjeuner. Les appareils n'étaient pas toujours prêts au moment critique - sujets manqués pour cette raison, la meilleure lumière du jour perdue; retards. 
Arrivé sur les lieux, en présence du sujet ou des personnes à consulter, les préliminaires doivent être brefs ; il faut aller au but. Les longues conversations sont en pure perte. On m'a fait souvent fait remarquer - à moi qui pratique cette économie - comme je prends peu de temps à me mettre à la besogne. Je cherche des chansons, je demande aux gens : " Savez-vous telle complainte? Tel tortillon ? Connaissez-vous le Roi Renaud, Là-haut sur ces montagnes ? On répond oui ou non. Si c'est oui, qui ici peut la chanter? - le grand-père - Très bien, chantez-la ! » Le cahier est prêt, aussi le stylo. Le vieillard chante, ses paroles sont prises à la sténographie. «Un autre ici sait une autre chanson. Laquelle ? Est-elle bonne ? Chantez-la!» Et puis d'autres encore. Lorsqu'il y en a 10 ou 15 de prises ainsi, voilà le phonographe. En quelques tours de main, il est placé, tous près. Quelqu'un est placé devant le cornet. Tout le monde est curieux, intéressé. Dans une heure ou deux, plusieurs bonnes chansons -10 ou 15 - sont déjà recueillies. On les fait écouter, pour vérifier si elles sont bien enregistrées, et pour susciter ou maintenir l'intérêt des chanteurs et de leur entourage.

Alors on peut s'arrêter et causer. Plus tard, vos hôtes d'occasion prennent le temps d'arrêter, de vous demander : " Mais d'où venez- vous ? Pourquoi prenez-vous ces vieilles choses ?» Et toutes sortes de questions. C'est le temps de répondre, de causer, de plaisanter.

Puis continuez la besogne aussi longtemps qu'il est bon. Si la chanson qu'on vous offre sort des livres, rejetez-la en disant : «Ah : non, c'est du neuf, ça sort des livres, d'un journal ; il ne faut que du vieux, appris des anciens, par cœur. » On saisit votre idée et on cherche à s'y conformer. La même règle de conduite pour les contes, les légendes, les recettes.

$\mathrm{Si}$ vous recherchez des tissus domestiques, des machines agricoles, des objets anciens, en entrant dans la maison, examinez aussitôt la bâtisse, demandez si elle ancienne, qui l'a bâtie ? Comment est-elle bâtie ? Le toit est-il le même qu'au commencement ? L'a-t-on remplacé depuis ? Venez voir, monsieur ! Venez au grenier, à la cave, à la dépense. Aussitôt arrivé au grenier, vous voyez des vieux meubles, une quantité d'outils curieux, qu'il serait bon d'acheter pour un musée ; il y a des coffres remplis. La maîtresse de la maison vous montre du tissage dont elle est fière. Tout à coup, dans un coffre, vous apercevez un objet curieux, un souvenir. Qu'est-ce que c'est ça ? Si ça vaut la peine de le photographier, mettez-le de côté. Si c'est après le dîner, les hommes du champ ont été, un moment, attirés à venir voir ; après quelque temps, ils se disent entre eux : « Retournons à l'ouvrage ! Les femmes peuvent voir au reste ; la maison, c'est leur affaire. » Et de part et d'autre, le travail continue.

Tout ça, c'est du doigté, de la technique. Vous allez vite en besogne. Les résultats viennent comme ils le doivent. Les gens mêmes que vous consultez 
vous estiment davantage. Ils voient que vous vous y entendez. Et ils se prêtent volontiers à votre direction, d'autant plus qu'ils sont surpris à la fin du jour ou de la visite, que vous les récompensiez en argent, pour le temps soustrait à leur routine domestique, ou en achetant des objets intéressants pour un prix qui leur paraît approprié.

\section{La concentration de l'attention}

La concentration de l'attention du chercheur est nécessaire. Sans l'habitude de la concentration; l'attention se disperse sur toutes sortes de sujets, en pure perte de temps. Le décousu se met de la partie ; on ne sait plus où l'on en est rendu. Ne cherchez pas à exécuter deux choses à la fois.

Il faut que l'oreille soit sensible, prenante, « éliminante » aussi. Dans la maison quelquefois remplie de monde qui jase, rit, claque les portes, on doit être capable d'entendre promptement, sans interruption, sans faire répéter, sans trop questionner, tout ce qui est chanté, raconté. Quitte à faire une petite croix en marge et à revenir plus tard compléter le renseignement.

L'habileté que l'expérience peut engendrer dans ce travail est étonnante. Les amateurs ou les curieux autour de vous sont portés à disperser leur attention. Vous ne songez qu'à une chose, en ligne droite. Ainsi vous polarisez le travail et vous vous assurez une bonne cueillette.

\section{Des sujets particuliers demandent une connaissance et une technique spéciales}

\section{A. Cueillette des textes}

1. Textes de contes, légendes, anecdotes formels, en français ou en anglais. Il y a peu de textes parlés qui soient absolument définitifs ; ils sont d'ordinaire sujets à de l'improvisation, ou à l'inspiration du moment. C'est pourquoi il faut, le moins qu'on peut, distraire le conteur. Les textes doivent être chantés ou dits au chercheur ; ils sont recueillis, si c'est possible, en sténographie, sinon, à l'écriture ordinaire ; il est bon de recueillir quelques spécimens au phonographe. Le moins d'interruption, le mieux. Explications après coup.

2. Textes équivalents en langue indienne. Comment les recueillir. La meilleure manière, pour un linguiste, est d'écrire les textes sous la dictée, en écriture phonétique, en la présence d'un interprète qui connaît le mieux possible les deux langues. Ici l'informateur et le chercheur sont les deux principaux acteurs. Une fois le texte écrit au complet, l'interprète entre dans son rôle ; pour la traduction littérale et grammaticale du texte, l'interprète et le chercheur deviennent les principaux acteurs - l'informateur est toujours 
là, prêt à fournir les explications requises. Enfin l'interprète donne une traduction libre, à grands traits, des phrases complètes. L'informateur peut être son propre interprète lorsqu'il parle les deux langues.

Qualités d'un bon interprète : intelligence, bonne connaissance des deux langues et entendement des procédés grammaticaux, intérêt personnel dans le travail. Mes meilleurs interprètes : William Beynon et Charles Barton, chez les Tsimsyans ; Allen Johnson, chez les Wyandots. Un autre type : Mrs Constance Cox, à Hazelton - l'incident Cox-Watserh, où l'informateur se plaint que les questions du chercheur «got his goat 》- le tracassaient, cela surtout parce que l'interprète n'avait pas le don de faire comprendre le but des questions, auxquelles la réponse était nécessaire ; et l'informateur luimême n'était pas bien imbu de son sujet - il savait machinalement les textes de ses chansons sans être bien renseigné sur leur sens et leur fonction. S'il n'y a pas lieu de recueillir les textes, ce qui est long et assez ardu, on prend par écrit le récit tel que l'interprète le transpose au fur et à mesure ; cela est beaucoup moins satisfaisant, le langage est plus pauvre.

3. Textes de chansons, des Sauvages ou des Blancs. Ces textes sont toujours fixes. Ils sont recueillis textuellement à la sténographie, si c'est possible, pour le français, ou en phonétique pour les langues étrangères. Les mêmes procédés servent pour recueillir les contes et les textes parlés. L'inconvénient d'interrompre un chanteur qui donne son texte ; cela lui fait perdre le fil de la récitation. En français, il est bon de recueillir bon nombre de textes de chansons, pendant que l'informateur les chante ; ensuite, de recueillir sur le phonographe, la mélodie de chaque chanson déjà dictée, deux couplets d'ordinaire suffisent. Différences dans la mélodie suivant les couplets ${ }^{21}$.

\section{B. Cueillette des mélodies, des danses}

1. Numérotage provisoire des mélodies ; cartes index. Plus tard, au Musée, des numéros définitifs seront donnés, numéros qui se conforment à un système d'ensemble assez compliqué. (Expérience à faire, ici, à l'aide du phonographe.)

2. Danses. Airs de danse, au violon, chantés, etc., recueillis sur le phonographe. Explication donnée des danses et de la manière de les exécuter. Il est bon de filmer ces danses, lorsque cela est possible. Signes graphiques conventionnels en usage parmi ceux qui se font une spécialité de cette étude. The English Folk Dance Society, dont les quartiers généraux sont à Cecil

21. Cette méthode de cueillette tenait probablement du fait que les cylindres de cire ne permettaient, selon les cas, que 2 ou 4 minutes d'enregistrement. 
Sharpe House, à Londres ; et ses succursales en Amérique. Collection ancienne de Mahé pour les mélodies de danses de la Bretagne (dans Mélusine).

\section{C. Étude des arts manuels, des procédés}

Étude des procédés de l'ethnobotanique, des teintures, de la technologie, des arts manuels, des recettes, etc. Ici le chercheur doit questionner ses informateurs suivant les connaissances qu'ils possèdent; il doit bien fouiller le sujet jusqu'à ce que tout soit tiré au clair. Ses notes doivent être prises sur le champ, le plus possible suivant les termes qu'il entend et qu'il note. Souvent il se glisse des mots rares et anciens dans ce langage ad hoc. Cela sert, en même temps, d'étude linguistique, qui enrichit le glossaire. Les cas de la " pêche à l'anguille » sur le Saint-Laurent; de la construction d'une « barque pêcheuse ", à Gaspé. Il est bon de recueillir des spécimens au point de vue d'en former une collection complète, et aussi de photographier sur place la reproduction des procédés, ou de bons exemples fournis. Dans le cas de l'ethnobotanique et de plantes servant aux teintures, il est nécessaire de recueillir les plantes, pour qu'elles soient plus tard identifiées, au Musée, par un botaniste. Un botaniste seul (comme M. Jacques Rousseau et ses élèves, qui ont entrepris de ces études) peut se passer de recueillir les plantes spécimens, vu qu'il les identifie correctement sur les lieux.

Dans les recettes, il faut recueillir les quantités de chaque élément qui entre dans la composition de l'ensemble. Les femmes de la campagne sont portées à être vagues sur ce point ${ }^{22}$.

\section{L'étude du langage}

Pour l'entreprendre et la pousser à fond, il faut être linguiste. Une oreille sensible et fidèle est nécessaire. Aussi faut-il acquérir la connaissance d'alphabets phonétiques pour un usage particulier. L'alphabet Rousselot, en France, surtout pour les dialectes français. L'alphabet du Smithsonian pour les langues américaines. Le Dr Franz Boas, de Columbia University, avait assez transformé cet alphabet, pour son usage particulier. Et Boas fut longtemps l'arbitre dans cette science, où il forma plusieurs bons élèves, entre autres le Dr Sapir. Sapir et moi-même avons transformé l'alphabet du Dr Boas, pour l'adapter à notre usage, au Canada. C'est cet alphabet qui a servi dans tous nos travaux.

L'appareil Rousselot pour recueillir les sons à l'aiguille - la vibration causée par les sons démarquée par une aiguille sur un rouleau de papier

22. En 1936, Marius Barbeau avait ainsi recueilli des centaines de recettes culinaires traditionnelles qu'il espérait publier après en avoir fait valider les ingrédients et leur proportion. Malgré la préparation d'un manuscrit, l'ouvrage toujours inédit demeure dans ses archives au Musée canadien des civilisations. 
fumé - sert en France ; le Dr Pliny Goddard s'en est servi chez les DénéAthapascans, mais il n'a pas tiré d'utiles résultats de son essai. L'emploi, chez les Sauvages, de cette machine compliquée et coûteuse n'a pas produit de résultats.

Méthode de Mademoiselle Sturm, linguiste française en Amérique. Sa méthode d'enregistrer les sons (surtout les voyelles), à Sainte-Famille, île d'Orléans. Ses difficultés.

L'étude de la phonétique française, au Canada, est encore à faire, surtout étant donné qu'elle est accomplie en France. On parle souvent des sources du français en notre pays, mais on n'a pas encore étudié à fond la linguistique laurentienne.

Le cas très significatif d'intonation (tone pitch) dans les dialectes des Dénés (Athapascans) ; singularité linguistique découverte par le Dr Sapir, qui relie ce langage américain aux langues de l'Asie (en particulier, au chinois).

Comment les alphabets des anciens missionnaires restaient incomplets, insuffisants - à défaut d'alphabet linguistique.

\section{E. Étude des beaux-arts et des arts utilitaires}

1. Orfèvrerie; vases sacrés, reliquaires, statues en argent; ustensiles domestiques; argenterie de la traite des fourrures. Orfèvrerie des Indiens. Manière de l'étudier ; les marques ; les mesures ; la photographie.

2. La broderie, la dentelle; ornements d'église dans les monastères. Où l'on trouve les meilleurs spécimens. Histoire de cet art (Saintes artisanes I, les Brodeuses) $)^{23}$. L'art de la broderie, chez les Sauvages, découle de l'art français colonial. La plus ancienne collection de la broderie chez les Sauvages - la collection du Dauphin de France, maintenant au Musée de l'Homme, à Paris. Comment observer et étudier ces reliques. Photographie ; analyse des matériaux. Instruments - métiers à dentelle chez les Ursulines de Québec.

\section{Sculpture}

\section{a. Sculpture laurentienne sur bois}

La sculpture au Canada découle de l'art français ancien ; son entrée au pays date des premiers temps. Les maîtres canadiens. Leur style variait au cours des générations. D'abord, jusque vers 1700 , on sculptait le bois dur, comme en France ; on passa ici, au bois mou, surtout au pin. On se servit plus tard du noyer (dur, tendre), aussi du bois blanc. Habitude de dorer et de carner les

23. Barbeau fait référence à son ouvrage publié chez Fides dans les Cahiers d'art Arca en 1943. Un second volume, intitulé Saintes artisanes II, Mille petites adresses, sera par la suite publié en 1946. 
statues. Art spécial des monastères, à commencer par les Ursulines. Diffusion de cet art, dans les monastères. À quoi on reconnaît les maîtres des diverses époques. Manière d'étudier ces pièces : mesures ; quelle sorte de bois, comment il est décoré.

\section{b. Sculpture sur bois des Indiens}

Les totems de la côte Nord-Ouest. C'est mon étude particulière. Ma principale découverte, à ce sujet, est que l'art des grands totems ne date que du XIX ${ }^{e}$ siècle. Sculpture sur bois des autres Sauvages, de peu d'importance, par exemple, pour les masques et les cuillers des Iroquois.

c. Sculpture en argilite des Hä̈das, des îles de la Reine Charlotte, près d'Alaska.

d. Sculpture sur ivoire. Défenses de morse, de mammouth (préhistorique) chez les Esquimaux, ainsi que sur la côte nord-ouest. Art moderne, dû à l'influence des chasseurs de baleines de Naraganset et de Gaspé.

\section{Architecture}

Elle requiert la connaissance du mesurage en architecture et de la manière de dresser les plans. Son étude, dans Québec, par l'école d'architecture de McGill - le professeur Ramsay Traquair et ses élèves. J'y ai moi-même contribué en simple auxiliaire et historiographe ${ }^{24}$. Feu Gordon Neilson et le professeur [Edward Robert] Adair y ont contribué. M. Gérard Morisset s'y intéresse aussi, mais son intérêt porte surtout sur la peinture, la sculpture et l'orfèvrerie.

Dans l'architecture domestique, il faut observer les modes de construction : en pierres, en bois. Plans des maisons, de leur toit, de leurs cheminées. Cas d'études précipitées, mal faites, par des amateurs, à Québec. Étude bien exécutée des maisons françaises en Louisiane et en Missouri : à l'initiative des Parcs nationaux de Washington.

Le type le plus intéressant est la construction en bois. Comment observer : « colombage », poteaux sur sole - ses variations intéressantes, poteaux en terre; la «tête de chien » (le type «log cabin» américain d'origine scandinave).

24. En 1925, Barbeau avait invité le professeur Traquair à venir le rejoindre à l'île d'Orléans pour y faire l'étude de l'architecture ancienne. Il en résulta plusieurs articles dans The Journal of Royal Architectural Institute of Canada où Barbeau fut coauteur : "The Church of Saint Famille, Island of Orleans, Que. »; «The Church of St. François de Sales, Island of Orleans, Que. »; « The Church of Saint Pierre, Island of Orleans, Quebec»; «The Church of Saint Jean, Island of Orleans, Quebec». 
Les types britanniques : «frame houses »; leur apparition dans diverses parties du pays, sur les frontières de Québec. Les types canadiens dans le Nord-Ouest à l'usage des compagnies de fourrure.

Types de maisons indiennes - iroquois-huron, algonkin, athapascan, côte nord-ouest, Esquimaux.

\section{F. Collection de spécimens}

À l'emploi d'un musée, il est nécessaire de faire la collection de spécimens de toutes sortes, suivant les circonstances. Méthode à suivre pour les achats ; notes à prendre, qui sont aussi importantes que les spécimens eux-mêmes. Les collectionneurs de curiosités, les amateurs, les marchands d'antiquités, négligent toujours ce côté, et détruisent, en quelque sorte, ce à quoi ils touchent.

Numérotage et étiquetage des spécimens d'abord provisoirement, ensuite, au Musée, définitivement, suivant le système adopté. Empaquetage, transport. Il faut tenir compte de la pratique. Emmagasinage et conservation dans les Musées. Système suivi. Danger des mites, des insectes, de la lumière.

\section{G. Études d'archives}

Exemples : a. - Archives de paroisses canadiennes ; b. - Archives de monastères, de séminaires, de collèges ; c. - Archives régulières, comme celles du Séminaire de Québec, de Saint-Sulpice ou des gouvernements. Catalogue fait ou à faire; analyse des pièces; reproduction exacte de leur titre ; indication de leur âge, dimensions, état de conservation, etc. Sources précieuses à découvrir.

\section{H. Connaissance de la littérature}

C'est là une connaissance qu'il faut avoir au bout de ses doigts : pour l'étude du folklore, de l'ethnographie, en tout ce qui jette de la lumière sur les sujets adoptés et également dans les études comparées qui s'imposent, surtout en folklore. 